\title{
Impact of Diabetes Type 2 in Adults on Autonomic Modulation at Rest and in Response to the Active Orthostatic Test
}

\author{
Vinodh R S ${ }^{1}$, Veerendra Kamath ${ }^{2}$, Shabina C, ${ }^{3}$ Shankarappa C ${ }^{4}$ \\ ${ }^{1}$ Associate Professor, ${ }^{2}$ Junior Research Scholar, ${ }^{3}$ AssistantProfessor, ${ }^{4}$ Prof \& HOD, Dept. of Physiology, \\ Vydehi Institute of Medical Sciences and Research Center, \#82, EPIPArea, Nallurahalli, Whitefield, Bangalore
}

\begin{abstract}
Background: Cardiovascular autonomic neuropathy Is one of the most common complications of Diabetes Mellitus Type 2(DM2). Heart rate variability (HRV \} Is a noninvasive measure of cardiac autonomicmodulation. Reduced HRVIs an Independent cardiovascula rrisk factor and has been proposed as amarker of increased risk of mortality. Very few studies have measured Changesin HRV in DM2 in India.
\end{abstract}

Objectives: To analyze the autonomic modulation responses In DM2 patients by means of HRV indices.

Methodology: Across-sectional study of 20 Type 2 Diabetic mellitus from age groups 30S0 years and age and gender matchenon-diabetic subjects as controls. Subjects satisfying in clusion and exclusion criteria and gave written informed consent were randomly invited to take partin the study. Initial assessment was done for HTN, hyperlipidemia, smoking, cardiovascular disease, family

H/o DM2, FBS >130 mg/di, Body mass index (BMI) $>25 \mathrm{~kg} / \mathrm{m}, \mathrm{DM} 2$, duration of disease, and Heart rate.

Bloodpressure: measure dmercury sphygmomanometer aftera5-minrest. Electrocardiogram wasrecordedusingPower Lab• (ADlnstruments). The time domain, frequencydomainvariables, and nonli near parameters was meas ured from ECGusing Lab Chart• software.

Results and Conclusion : Time domain Variablesnamely AverageRR, SDRR,SDARR,rMSSD andPNNS0(\%) and Frequency domain Variables; High frequency (HF) power, Low frequency (LF) power and Very Lowfrequency (LF)power Non-Linear Variables; S01and SD2 were reuced in patients with diabetes Mellitus compared to normal control but was not statistically significant. One of the reasons couldbesmallsamplesize. Study should be repeated in large sample size.

Key Words : Heart Rate Variability, Autonomic Dysfunction, Type 2 Diabetic Mellitus

\section{Introduction}

Diabetes mellitus is one of the most common Non-Communicable Diseases and, as well as the aging process, may influence the autonomic nervous system (ANS), leading to a poor autonomic control of heart
1,2. In subjects with diabetes mellitus, damage to both parasympathetic and sympathetic fibers innervating the cardiovascular system produces the cardiovascular autonomic neuropathy (CAN). CAN clearly entails an increase in mortality and an acceleration of other 
micro vascular complications ${ }^{3}$. Among the most common complications, it is highlighted the diabetic autonomic neuropathy which is poorly recognized and understood despite its significant effects on several organs and systems ${ }^{4,5}$. Heart-rate variability is a measure of cardiac autonomic function ${ }^{6}$. The heart rate variability (HRV) was proved to be a noninvasive tool as valuable clinical evidence for the prognosis of cardiovascular events and several disorders. Reduced $\mathrm{HRV}$ is an independent cardiovascular risk factor ${ }^{7}$, ${ }^{8}$. At an early-stage autonomic dysfunction may be asymptomatic or mildly symptomatic. Symptomatic autonomic neuropathy carries worst prognosis, so early diagnosis is essential for maximum benefit more so in Diabetes ${ }^{9}$. Also, very few studies have measured changes in Heart Rate Variability in type 2 diabetes mellitus in India. Thus, analysis of HRV associated with the autonomic active orthostatic test is important in South Indian population. Hence in the present study our aim is to analyze the autonomic modulation responses induced by the implementation of the active orthostatic test, in adults with DM2, and study the autonomic modulation by means of HRV indices.

\section{Objectives}

1. To determine HRV in type 2 Diabetics Mellitus.

2. To determine Autonomic modulation responses induced by the implementation of active orthostatic tests in adults with type 2 diabetics mellitus.

\section{Material and Methods}

A cross sectional study of 20 type 2 Diabetes Mellitus patients from age groups 30-50 years and 20 age and gender matched non-diabetic subjects as controls.

\section{Inclusion criteria}

Patients with Diabetes Mellitus type 2 minimum disease duration $>2$ year with controlled glycemic status defined as (1) glycosylated hemoglobin $(\mathrm{HbA} 1 \mathrm{c})<7 \mathrm{mg} \%$ (2) $\mathrm{FBS}<126 \mathrm{mg} \%$ and (3) post prandial blood sugar $<180 \mathrm{mg} \%$ (ADA guidelines)

\section{On regular hypoglycemic medication but not} insulin

- Patients who are ready to give written informed consent

\section{Exclusion criteria}

- Those on irregular treatment, newlydiagnosed ( $<6$ months)

Previous h/o neurological or cardiovascular intervention, onpacemaker, on drugs directly affect ANS, arrhythmia

Initial assessment was done for Hypertension, Hyperlipidemia, Smoking, Cardiovasculardisease, family $\mathrm{h} / \mathrm{o}$ diabetes mellitus, FBS $>130 \mathrm{mg} / \mathrm{dl}$, BMI $>25 \mathrm{~kg} / \mathrm{m} 2$, duration of diabetes mellitus and Heart rate.

Assessment of HRV was carried out between 8.30 and 12.00 am in a separate examination room. Patients were requested to avoid coffee, tea, cola drinks, and smoking for $12 \mathrm{~h}$ and alcoholic beverages for $24 \mathrm{~h}$ before the procedure

Blood pressure was measured by mercury sphygmomanometer after a 5 min rest.

Electrocardiogram was recorded after supine rest for at least 5 min with subject being in supine position and breathing freely using power lab (AD Instruments). The ECG recording from the precordial leads was transferred online to a microcomputer for the analysis of HRV.

Heart rate variability was measured from ECG using Ad instrumentslab chart software

Autonomic Test—Active Orthostatic Test 
To perform the active orthostatic test the subjects will be instructed to remain lying at rest for 30 minutes, after which they will stand up (3 to $4 \mathrm{~s}$ ), remaining in a standing position for 10 minutes. The subjects will be monitored throughout the period. The RR interval sequence with greater stability was selected from the initial rest period and during the maneuver for each subject

\section{Statistical Analysis}

The statistical significance of differences in the mean distribution of various parameters among various subgroups is done by Mann-Whitney test or unpaired Student's $t$-test for quantitative data.

Time Domain Variables

- Average RR: Mean RR Interval

- SDRR: Standard deviation of R-R Interval
- SDARR: Standard deviation of average R-R Interval

- rMSSD: Root mean square of successive RR interval differences

- $\quad$ PNN50(\%) time domain heart rate variability

Frequency Domain Variables

- $\quad$ High frequency (HF) power

- Low frequency (LF) power

- $\quad$ Very Low frequency (LF) power

Non-Linear Variables

- $\mathrm{SD} 1$

- $\quad \mathrm{SD} 2$

\section{Results}

FIGURE 1

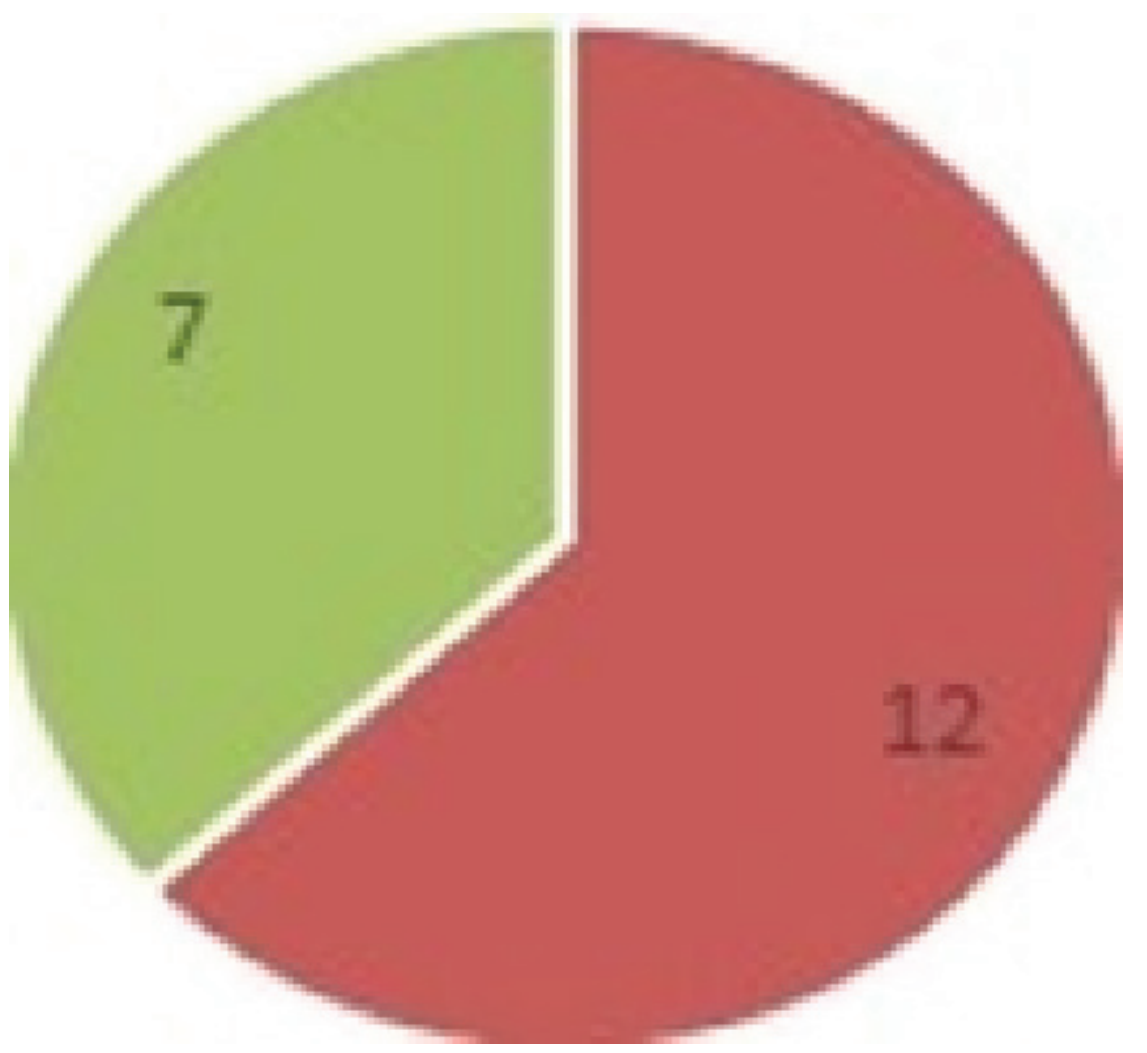




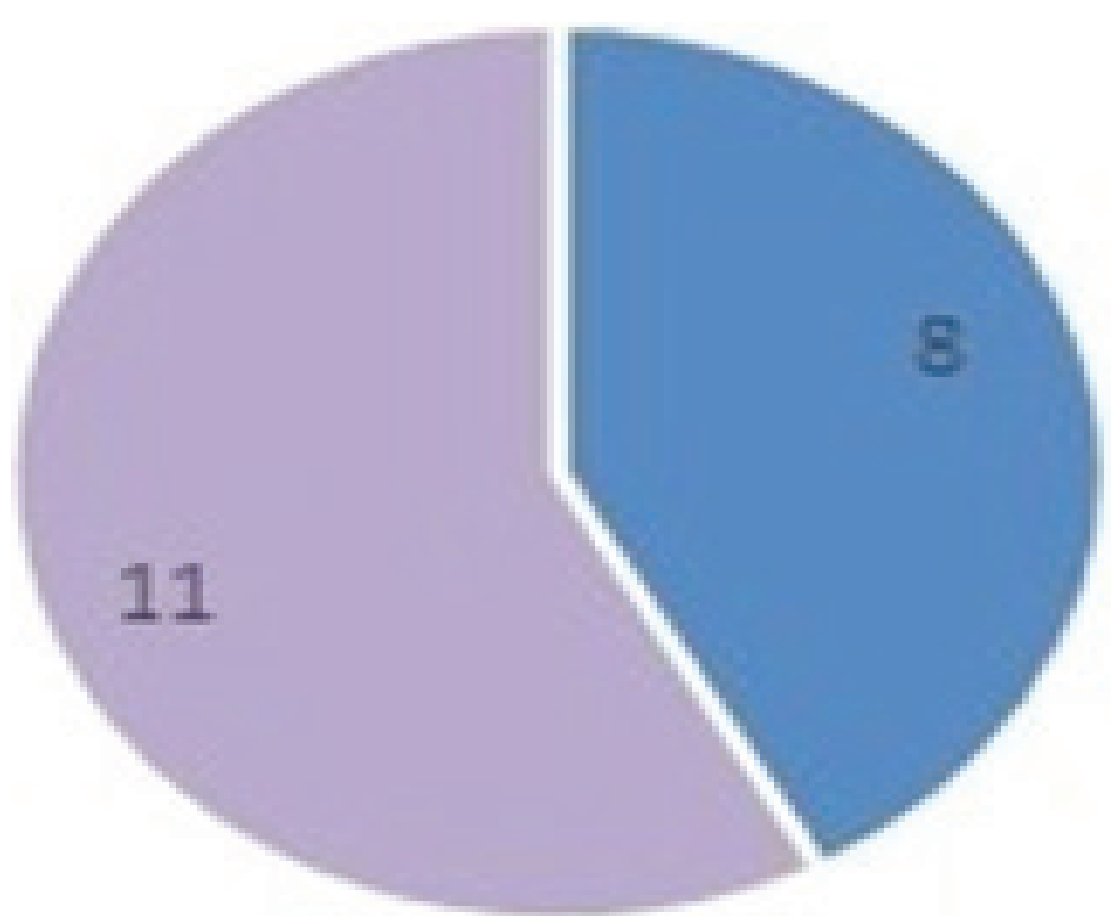

DIAGRAM 2 SHOWING DISTRIBUTION OF SUBJECTS INTO DIABETICS AND NON-DIABETICS

Table-1: Comparison of Time, Frequency Domain and nonlinear variables in diabetic and non-diabetic patient

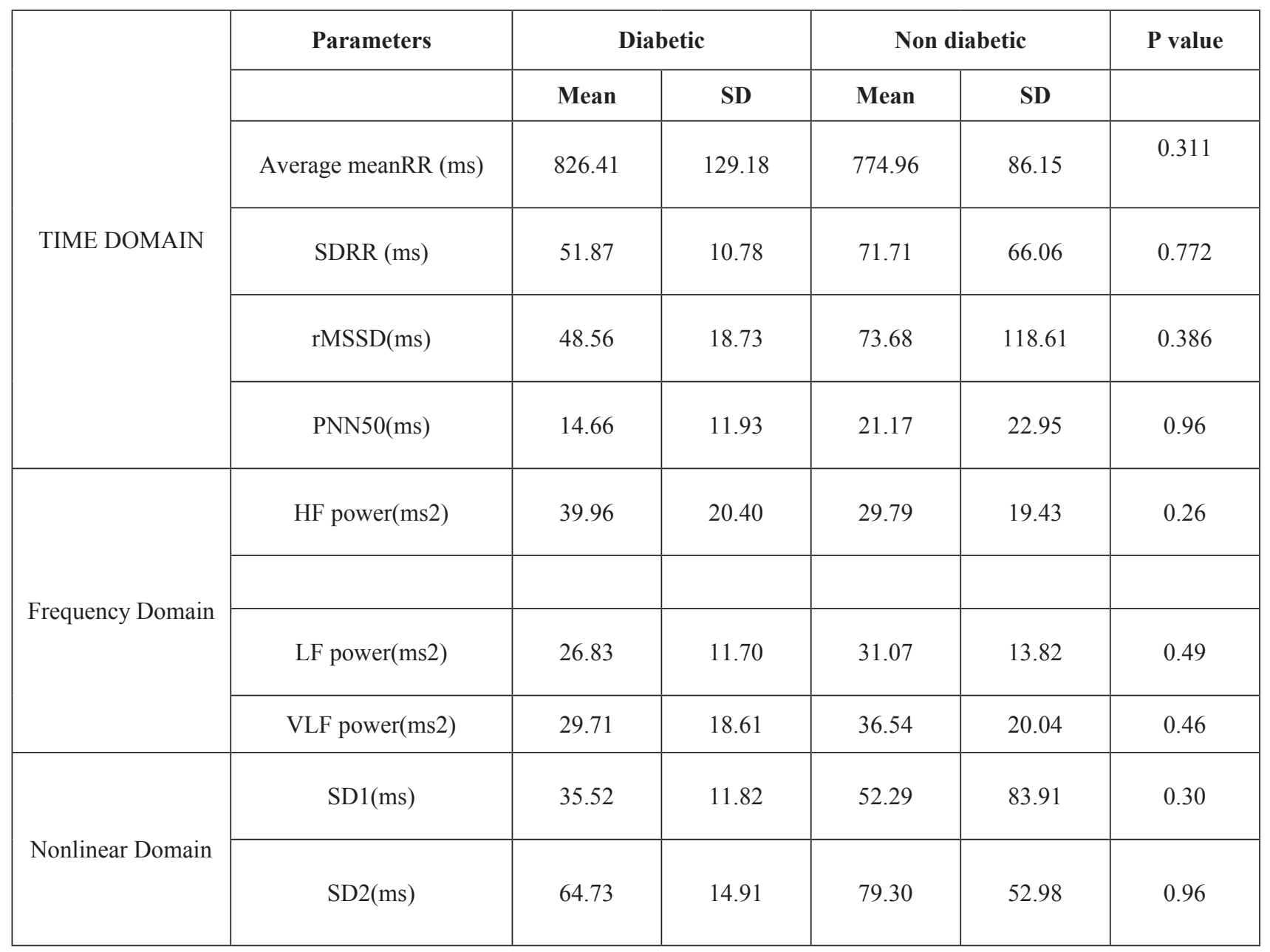




\section{Discussion}

Heart rate variability (HRV) asserts the variations of instantaneous $\mathrm{HR}$ as well as RR intervals. Decreased HRV is a recognized vital autonomous risk element for greater mortality and sudden cardiac death (SCD) in cardiovascular disease and healthy populations ${ }^{10}$. In about half of the patients, diabetesmanifests as autonomic neuropathy leading to autonomic imbalance which is a bad prognostic factor ${ }^{11,12}$. In a latest meta-analysis including 15 researches in diabetic individuals, cardiac autonomic neuropathy established an appreciable association with mortality when abnormal values of two or more indices of HRV expressed autonomic imbalance. Diabetes is known to reduce HR variability. Variations in HRV had been observed in different studies on diabetic patients among different populations based on the variations in their autonomy.In diabetic individuals with associated neuropathy, decreased value of SDNN appears to bear negative prognostic value and herald the manifestation of autonomic neuropathy. The mechanism of diabetic neuropathy is not very comprehensible, although it might be correlated to the disturbance of metabolism and autonomic nerves malnutrition ${ }^{13}$. In our study Non-Linear Variables andSD2werereduced inpatientswithdiabetes Mellitus compared to normal control but was not statisticallyy significant. Thereason could be small sample size. Study should berepeated in large sample size involving various complications like ischemia and foot ulcers to name a few.

\section{Conclusion}

TIPNN50 medomain Variables namely Average RR,SDRR, SDARR, RMSSD and (\%) and Frequency domain variables, High frequency (HF) power, Low frequency (LF) power and Very Low frequency (LF) power

Non-Linear Variables'andSD2 were reduced in patient swith diabetes Mellitus compared to normal control but was not statisticallyy significant. Thereason could be small sample size. Study shoud be repeated in large sample size

Ethical Clearance- Taken from Vydehi Institutional Ethics Committee (VIEC)

\section{Source of Funding- Self}

\section{Conflict of Interest - Nil}

\section{References}

1. A. Voss, A. Heitmann, R. Schroeder, A. Peters, S. Perz Short-term heart rate variability - age dependence in healthy subjects. Physiol Meas, 33 (2012), pp. 1289-1311

2. G. Danaei, M.M. Finucane, Y. Lu, G.M. Singh, M.J. Cowan, C.J. Paciorek, et al. National, regional, and global trends in fasting plasma glucose and diabetes prevalence since 1980: systematic analysis of health examination surveys and epidemiological studies with 370 country-years and 2.7 million participants Lancet, 378 (2011), pp. 31-40

3. Vinik AI, Maser RE, Mitchell BD \& Freeman R. Diabetic autonomic neuropathy. Diabetes Care $2003261553-1579$.

4. Vinik AI, Erbas T. Recognizing and treating diabetic autonomic neuropathy. Cleve Clin J Med. 2001; 68(11): 928-930.

5. Vinik AI, Freeman R, Erbas T. Diabetic autonomic neuropathy. Semin Neurol. 2003; 23(04): 365-372.

6. Task Force of the European Society of Cardiology and the North American Society of Pacing and Electrophysiology: Heart rate variability: standards of measurement, physiological, interpretation, and clinical use.Eur Heart J17: 354-381, 1996

7. R. E. Kleiger, P. K. Stein, and J. T. Bigger Jr., "Heart rate variability: measurement and clinical utility," Annals of Noninvasive Electrocardiology, vol. 10, no. 1, pp. 88-101, 2005.

8. S.Fenske, R.Pröbstle,F.Aueretal., "Comprehensive multilevel in vivo and in vitro analysis of heart rate fluctuations in mice by ECG telemetry and electrophysiology," Nature Protocols, vol. 11, no. 1, pp. 61-86, 2015 
9. Ghosh Asuthosh and Mukherjee Chandra Subhas. Evaluation of Autonomic Function in Diabetes mellitus. Ind. J. Physiol and Allied Sci. 1998; 52 (1): $28-34$

10. Mylonopoulou M, Tentolouris N, Antonopoulos S, Mikros S, Katsaros K, Melidonis A, et al. Heart rate variability in advanced chronic kidney disease with or without diabetes: midterm effects of the initiation of chronic haemodialysis. A. therapy. Nephrol Dial Transplant 2010; 25:3749-54.

11. Powers AC. Diabetes mellitus. In: Braunwald E, et al (Eds) Harisonova nacela interne medicine 2011; pp.2109-37. Debono M, Cachia E. The impact of
CardiovascularAutonomic Neuropathy in diabetes: Is it associated with left ventricular dysfunction? Autonomic Neuroscience: Basic and Clinical 2007; 132:1-7.

12. Debono M, Cachia E. The impact of Cardiovascular Autonomic Neuropathy in diabetes: Is it associated with left ventricular dysfunction? Autonomic Neuroscience: Basic and Clinical 2007; 132:1-7.

13. JH, McDougal DB, Hauhart RE, Schulz DW. Effects of acute, subacute and chronic diabetes on carbohydrate and energy metabolism in rat sciatic nerve. Relations to mechanisms of peripheral neuropathy. Diabetes 1995; 44:190-5 